\title{
Comparison of Limberg Flap and Karydakis Flap Surgery for the Treatment of Patients With Pilonidal Sinus Disease: A Single-Blinded Parallel Randomized Study
}

\author{
Mina Alvandipour ${ }^{1}$, Mohammad Sadegh Zamani ${ }^{1}$, Mojtaba Ghorbani ${ }^{1}$, Jamshid Yazdani Charati ${ }^{2}$, \\ Mohammad Yasin Karami ${ }^{3}$ \\ ${ }^{1}$ Department of Colorectal Surgery, Mazandaran University of Medical Sciences, Sari; ${ }^{2}$ Department of Biostatistics, Health Sciences Research \\ Center, Faculty of Health, Mazandaran University of Medical Sciences, Sari; ${ }^{3}$ Department of Surgery, Shiraz University of Medical Sciences, \\ Shiraz, Iran
}

Purpose: Pilonidal sinus disease is a common condition, which mostly affects young men. While various surgical techniques have been introduced for treating intergluteal pilonidal disease (IPD), controversies still exist regarding the best surgical approach. The purpose of this study was to compare the efficiency and the short-term outcomes of Limberg flap and Karydakis flap surgeries for the treatments of patients with IPD.

Methods: A total of 80 patients with IPD who had underwent either Karydakis flap (KF group: $\mathrm{n}=37$ ) or Limberg flap (LF group: $\mathrm{n}=27$ ) surgery between January 2015 and January 2016 at Imam Khomeini Hospital of Sari in the North of Iran were recruited for inclusion in this randomized, single-blind study.

Results: Compared to the KF group, the LF group showed faster complete wound healing, longer duration of surgery and hospital stay, larger wound size, and shorter period of incapacity for work. The overall patient satisfaction in the LF group was significantly higher than that in the KF group. The visual analogue scale score of pain was lower in the LF group than in the KF group. Also, the overall frequency of postoperative complications was higher in the KF group than in the LF group. Recurrence was reported in one patient from the KF group.

Conclusion: Given the lower rate of postoperative complications and greater cosmetic satisfaction of patients, the Limberg flap procedure should be selected, instead of the Karydakis flap procedure, as the standard technique for treating patients with IPD.

Keywords: Surgical flaps; Postoperative complication; Pilonidal sinus disease; Recurrence

\section{INTRODUCTION}

Intergluteal pilonidal disease (IPD) is an infection of the skin and subcutaneous tissues at or near the upper part of the natal cleft of the buttocks. Although pilonidal cavities are considered pseudo-

Received: February 22, 2018 - Accepted: September 27, 2018

Correspondence to: Mohammad Yasin Karami, M.D.

Department of Surgery, Shiraz University of Medical Sciences, Zand Ave, Shiraz, Iran

Tel: +989171800710, Fax: +987132331634, E-mail: karamiy@sums.ac.ir ORCID code: https://orcid.org/0000-0003-2307-5569

(C) 2019 The Korean Society of Coloproctology

This is an open-access article distributed under the terms of the Creative Commons Attribution NonCommercial License (http://creativecommons.org/licenses/by-nc/4.0) which permits unrestricted noncommercial use, distribution, and reproduction in any medium, provided the original work is properly cited. cysts, the sinus tracts may be epithelialized [1]. The clinical presentations are consistently variable, ranging from an asymptomatic pilonidal cavity or sinus to acute infection or chronic inflammation and drainage $[2,3]$. The intergluteal cleft is the groove between the buttocks, which extends from right below the sacrum to the perineum and occurs due to the anchoring of deep skin layers overlying the coccyx to the anococcygeal raphe.

The incidence of IPD is estimated at 26 cases per 100,000 population. The mean age at IPD onset is 19 years in women and 21 years in men. Generally, men are 2 to 4 times more prone to this disease $[1,4,5]$. The risk factors for IPD include obesity, local trauma or irritation, prolonged sitting, a deep natal cleft, and positive family history $[1,2,5]$. The exact mechanism of the progress of pilonidal disease is uncertain, although hair and inflammation are recognized as contributing factors $[6,7]$. 
IPD normally develops in individuals with deep natal clefts. As a person sits or bends, the natal cleft stretches, damaging or breaking the hair follicles and opening a pore or pit. The pores collect debris and serve as a fertile environment for hair roots shed from the buttocks to lodge and become embedded. Cavities may contain hair, debris, and granulation tissue. Once the pore becomes infected, an acute subcutaneous abscess develops [1, 8]. Physical examination in patients with IPD indicates one or more primary pores (pits) in the midline of the natal cleft and/or a painless sinus opening cephalad and slightly lateral to the cleft. In patients with acute or chronic diseases, a tender mass, sinusdrained mucus, purulent fluid, and/or bloody fluid can be identified. Complete removal of these substances is expected to result in definitive treatment. However, in practice, pilonidal cysts can recur following an extensive surgical resection of the affected area.

The mainstay of operative management for chronic or persistent IPD is en bloc excision of the entire pilonidal sinus and epithelialized tracts, using methylene blue to identify the involved sinus tracts down to the level of the sacrococcygeal fascia [1, 9-11]. Optimal closure of the wound following excision is debated. Primary closure can be accomplished by using either midline or off-midline techniques $[12,13]$, including the Z-plasty, V-Y advancement flap [14], and rhomboid (Limberg) flap [15] techniques.

Complex reconstructive operations using flaps are typically applied for patients with an extensive disease or those unresponsive to simpler surgeries (e.g., excision and midline closure with sutures) [4]. These reconstructive strategies allow for the excision of more involved tissues and are associated with reduced tension in the healing wound. In addition, these techniques facilitate wound closure lateral to the natal cleft, an area characteristically moist, hypoxic, and bacteria-laden [16-18]. The sutured off-midline wounds take significantly less time to heal and show lower rates of surgical site infection, recurrence, and overall complications, compared to sutured midline wounds [12].

While an off-midline approach seems optimal for closure, the optimal off-midline procedure has not yet been examined. The lateral advancing flap (modified Karydakis flap) [19] and the modified version of the rhomboid flap (Limberg flap) [20] are the two most common off-midline approaches. On the other hand, wound disruption, wound dehiscence, and complications, such as wound infection, subcutaneous fluid collection, and seroma formation, are still undetermined in the Iranian population. The aim of this study was to compare the Limberg flap and the Karydakis flap outcomes in the treatment of patients with IPD to identify the best approach for reducing health-system costs and patients' problems in Iran.

\section{METHODS}

\section{Patients}

This randomized, single-blind, clinical trial was performed during one year from January 2016 to September 2017 in Imam Kho- meini Hospital (Sari, Iran), which is affiliated with Mazandaran University of Medical Sciences. The study protocol was approved by both the Institutional Review Board and the Medical Ethics Committee of Mazandaran University of Medical Sciences. All the participants and the parents of adolescent patients gave their written informed consents before the study. This clinical trial was registered in the Iranian Registry of Clinical Trials (IRCT20141218020364N8).

The inclusion criteria were as follows: (1) patients with IPD who had been referred to Imam Khomeini Hospital and (2) whose ages ranged from 15 to 65 years. On the other hand, the exclusion criteria were (1) elderly patients with comorbid diseases, (2) scars from a previous pilonidal surgery, (3) abscess presentations, (4) diabetes mellitus, (5) immunodeficiency, (6) neurological disorders, (7) drug addiction or alcoholism, (8) American Society of Anesthesiologists physical status classification grade III-IV, (9) age $<15$ years or $>65$ years, and (10) orifice located more than 3 $\mathrm{cm}$ from the sinus center.

A total of 98 patients were diagnosed with chronic IPD at the hospital through physical examination. Because 26 patients did not meet the inclusion criteria and 20 patients refused to participate in the study, they were excluded, so 52 patients were finally recruited (Fig. 1). After the patients had been instructed about the clinical features of surgical procedures and written informed consents had been obtained, they were referred to the colorectal clinic for randomization. The patients were assigned to 2 groups, i.e., the Limberg flap (LF; 27 patients) and the Karydakis flap (KF; 37 patients) groups, by using a computer-based table of randomization to draw sealed envelopes randomly.

In this single-blind study, the participants were familiar with two common types of IPD surgery, but due to skin wound dressing on the back, they were not aware of their treatment. In addition, the observer, who completed the questionnaire, was not familiar with the groups. Similarly, the data analyzer did not know the study groups. However, the main researcher (surgeon) had information about the groups, and both surgical procedures were performed by the same surgeon. The safety and data monitoring committee was also informed about the study groups.

\section{Intervention and follow-up}

For all patients, surgery was performed under spinal anesthesia in the prone position. The patients on the operating table were administered antibiotic prophylaxis using intravenous cefazolin (1 g). They were placed in the jack-knife position, which allowed a better view of the operation area. Both buttocks were retracted laterally by using sticky tape. The operating site was cleaned with $10 \%$ povidone iodine solution.

Surgery was performed in the LF group, as defined by Mentes et al. [21]. A rhomboid excision was performed with the lower edge $2 \mathrm{~cm}$ lateral to the midline and covered the entire area where the sinus extended. Hemostasis was accomplished by using electrocautery. In order to ensure tension-free repair, the flap was re- 


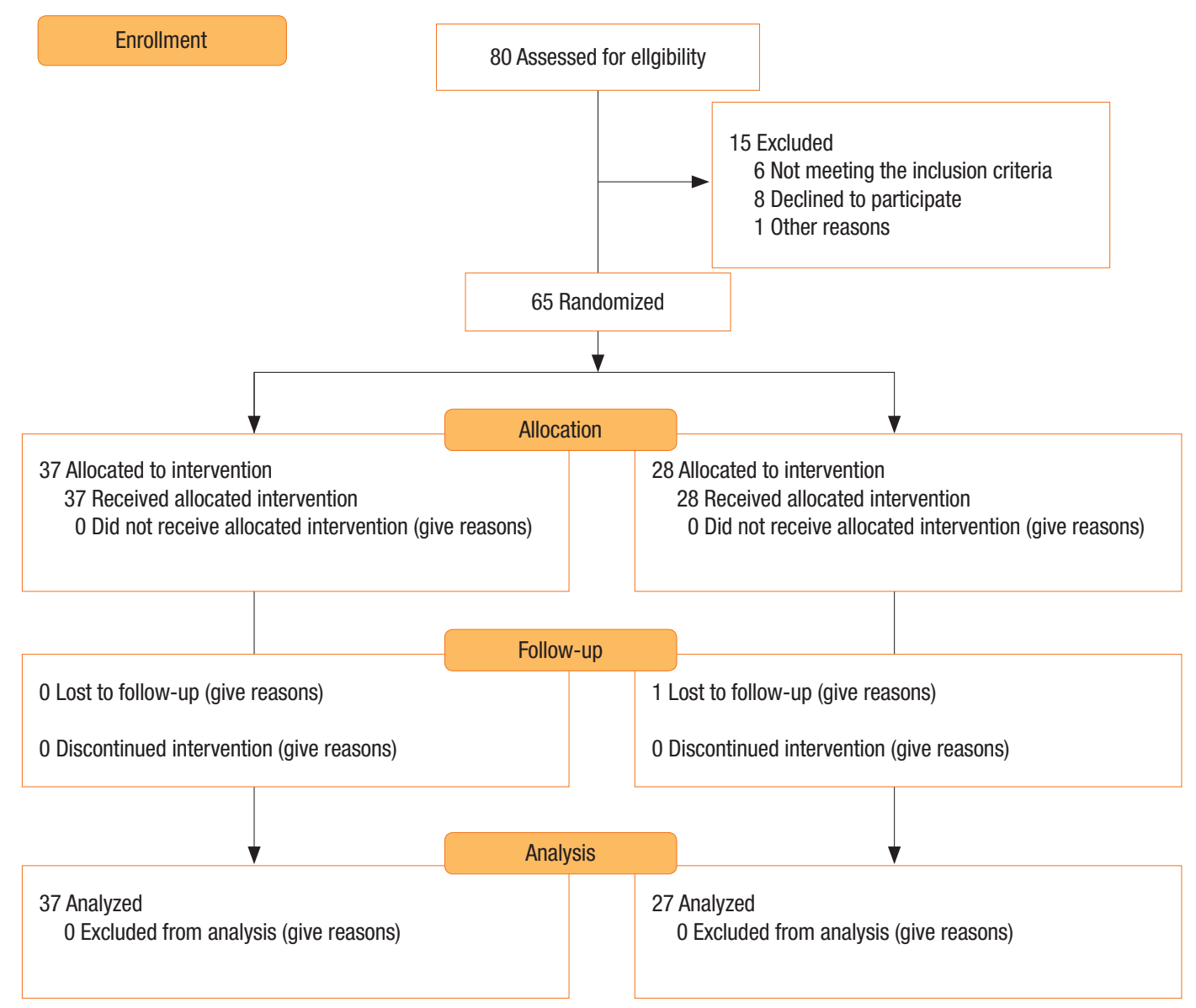

Fig. 1. The CONSORT (consolidated standards for reporting of trials) diagram showing the allocation process throughout the trial.

leased at the bottom involving the gluteal fascia and was then glided medially to cover the cavity defect. A suction drain was applied to the region in all patients. The subcutaneous tissue was closed with 2 fold 2/0 polyglactin suture (Ethicon US, LLC, Cincinnati, OH, USA), and the skin was closed with a $3 / 0$ polypropylene mattress suture (Ethicon US, LLC). The drain was removed when drainage fell below $40 \mathrm{~mL} /$ day.

Surgery was performed in the KF group, as described by Karydakis [22]. In this technique, an asymmetrical elliptic excision was made with the lower and upper edges located approximately $2 \mathrm{~cm}$ lateral to the natal cleft; all defective tissues were removed until the healthy borders had been reached. Afterwards, the medical wound edge was mobilized, and the flap was slid by suturing it to the fascia. The subcutaneous tissue was closed with 2 fold $2 / 0$ polyglactin suture, and the skin was closed with a 3/0 polypropylene mattress suture. In all patients, a suction drain was applied to the region; it was removed when drainage fell below $40 \mathrm{~mL} /$ day.

Data related to the patients' age, gender, operation type, operation duration, length of hospital stay, time of return to daily activities, preoperative infection (in the first week), hematoma (in the first week), seroma formation (in the first 2 to 3 weeks), and wound dehiscence (in the first 2 to 3 weeks) were collected. The visual analogue scale (VAS; $1-10$ items) was used in order to assess the patients' pain and cosmetic conditions. The recurrence rate was evaluated at 6 months postsurgery. Data were collected by examining the patients during postoperative dressing in the clinic. The patients' wounds were assessed daily, as daily dressing was needed during the patients' hospital stay. The patients were then assessed weekly in the first month following discharge from the hospital and at 3 months and 6 months following discharge from the hospital. For determining cosmetic satisfaction, we asked the patients the following question: "Please describe your satisfaction with the operation scar due to pilonidal sinus disease." Also, to assess pain, we asked the following question: "Do you feel pain while sitting on the operation site?" Please rate that pain on the VAS.

\section{Statistical analysis and sample size}

According to the following formula from a study by Bali et al. [23], and consider a drop-out rate of $23 \%$ a sample size of 26 subjects per group provided approximately a power of 0.9 for comparing the 2 treatment groups; 


$$
N=\frac{2 \times\left(z_{\frac{\alpha}{2}}+z_{\beta}\right)^{2} \times(1+(k-1) \rho)}{k \times\left[\frac{\left(\mu_{1}-\mu_{2}\right)}{\sigma}\right]^{2}}=20
$$

where $\alpha=0.05, \beta=0.1, \mu_{1}=2, \mu_{2}=4, \sigma=2, K=4, \rho=0.9$.

Statistical analysis was performed using IBM SPSS Statistics ver. 21.0 (IBM Co., Armonk, NY, USA). The mean and the standard deviation (SD) were measured for calculating numerical parameters while the frequency and the percentage were determined for categorical variables. In addition, the Mann-Whitney U-test was used to compare the data. For the comparison of categorical variables, cross-tabulation was used (chi-square - Fisher exact test). The results are presented as mean \pm SD or proportion, as appropriate. A 2-tailed P-value of less than 0.05 was considered statistically significant.

\section{RESULTS}

From the initial sample of 80 patients enrolled in this study, 15 were excluded. The study population comprised of 65 patients, including 31 female (47.69\%) and 34 male patients (52.30\%). One patient was lost during follow-up due to family problems. The final analysis was performed on 27 patients in the LF group and 37 patients in the KF group. The CONSORT (consolidated standards for reporting of trials) flow diagram of patients is shown in Fig. 1.
In the LF group, 18 out of 27 patients (66.7\%) were female while in the KF group, 21 out of 37 patients (56.8\%) were male. The sex distribution was not significantly different between the groups $(\mathrm{P}=$ 0.064).

The mean \pm SD of age in the KF group $(25.89 \pm 9.48$ years $)$ was lower than that in the LF group ( $34.19 \pm 10.47$ years), and this difference was statistically significant $(\mathrm{P}=0.002)$. The wound size ( $\mathrm{mm}$ ) was significantly larger in the LF group than in the KF group ( $28.11 \pm 8.48 \mathrm{~mm}$ vs. $23.84 \pm 8.44 \mathrm{~mm}$ ), although the complete wound healing time (days) was longer in the KF group than in the LF group ( $11.51 \pm 3.16$ days vs. $9.56 \pm 1.31$ days). The difference in surgery durations (minutes) between the groups was significant; it was, in fact, lower in the KF group $(29.15 \pm 7.69$ minutes vs. $23.03 \pm 6.06$ minutes, $\mathrm{P}<0.001$ ). Based on the findings, compared to the KF group, the length of hospital stay (days) in the LF group was longer ( $1.48 \pm 0.50$ days vs. $1.41 \pm 0.49$ days), but this difference was not significant $(\mathrm{P}=0.540)$. We found that postoperative duration of incapacity for work (days) was significantly longer in the KF group than in the LF group (11.59 \pm 3.44 days vs. $9.15 \pm 2.52$ days $)(P=0.005)$. Table 1 compares the study outcomes between the groups. The overall patient satisfaction in the LF group was significantly higher than it was in the KF group $(8.26 \pm 0.94$ vs. $7.62 \pm 1.32)$. In addition, the VAS score of pain was lower in the LF group than in the KF group (4.00 \pm 0.96 vs. $4.11 \pm 1.02)$. The median satisfaction scores were $9(37 \%)$ and 8 (28.1\%) in the LF and the KF groups, respectively $(\mathrm{P}=0.046)$.

Table 1. Clinical comparisons of the operative and the postoperative characteristics and of the complications between the 2 groups

\begin{tabular}{|c|c|c|c|}
\hline Variable & Limberg flap $(n=27)$ & Karydakis flap $(n=37)$ & P-value \\
\hline Sex, male : female & $18: 9$ & $16: 21$ & 0.064 \\
\hline Age (yr) & $34.19 \pm 10.47$ & $25.89 \pm 9.48$ & 0.002 \\
\hline Wound size (mm) & $28.11 \pm 8.48$ & $23.84 \pm 8.44$ & 0.039 \\
\hline Duration of incapacity for work (day) & $9.15 \pm 2.52$ & $11.59 \pm 3.44$ & 0.005 \\
\hline Duration of surgery (min) & $29.15 \pm 7.69$ & $23.03 \pm 6.06$ & 0.001 \\
\hline Length of hospital stay (day) & $1.48 \pm 0.50$ & $1.41 \pm 0.49$ & 0.540 \\
\hline Complete healing time (day) & $9.56 \pm 1.31$ & $11.51 \pm 3.16$ & 0.023 \\
\hline VAS pain score & $4.00 \pm 0.96$ & $4.11 \pm 1.02$ & 0.720 \\
\hline Patient satisfaction & $8.26 \pm 0.94$ & $7.62 \pm 1.32$ & 0.035 \\
\hline \multicolumn{4}{|l|}{ Complications } \\
\hline Fluid collection & $3(11.1)$ & $13(35.1)$ & 0.028 \\
\hline Wound infection & $1(3.7)$ & $3(8.1)$ & 0.472 \\
\hline Hematoma & $0(0)$ & $0(0)$ & 0.423 \\
\hline Bleeding & $0(0)$ & $0(0)$ & 0.341 \\
\hline Flap necrosis & $0(0)$ & $0(0)$ & 0.463 \\
\hline Wound dehiscence & $0(0)$ & $1(2.7)$ & 0.399 \\
\hline Recurrence & $0(0)$ & $1(2.7)$ & 0.389 \\
\hline
\end{tabular}

Values are presented as mean \pm standard deviation or number (\%). VAS, visual analogue scale. 
The median VAS scores were $12(44 \%)$ and $14(37.8 \%)$ in the LF and the KF groups, respectively $(\mathrm{P}=0.934)$; the maximum VAS score was 6 in both groups.

Postoperative complications were more common in the KF group than in the LF group $(\mathrm{P}<0.05)$. The most common postoperative complication in both groups was fluid collection. Also, fluid formation was more common in the KF group than in the LF group ( 3 vs. $13 ; \mathrm{P}=0.028$ ). No hematoma, bleeding, or flap necrosis occurred in either group. Wound infection was detected in 1 patient and 3 patients from the LF and the KF groups, respectively. Also, wound dehiscence and recurrence were found in one patient from the KF group.

\section{DISCUSSION}

A pilonidal cyst or pilonidal sinus is a small skin cavity with hair in the lower back. During World War II, this disease was known as the Jeep driver's disease. It appears as a small skin orifice and does not contain many intricate hair twists in some cases. This disease is more common in the sacrococcygeal region among white adult men. The highest age range is $16-26$ years, and cyst infection usually starts in early adulthood (18-40 years) [24-27]. Gurer et al. [28] and Bali et al. [23] reported mean ages of 25.5 and 24 years, respectively; in those studies, $95 \%$ of the patients were male. In our study, the mean age of the patients was 29 years, and $53 \%$ of the patients were male.

Today, considering the importance of time and rapid return to work and life activities, the off-midline approach is recognized as the best surgical technique, which can lead to full recovery within 1-12 days. The ultimate goal of treatment is improved wound healing, low relapse rate, short length of hospitalization, fewer complications, and high patient satisfaction. The results of the present study showed that the mean total recovery time after surgery was 9.51 days for the Limberg flap technique and 11.51 days for the Karydakis flap technique. In similar studies, such as the one performed by Bali et al. [23], duration of recovery was 22.12 days, and the full recovery time was 24.08 days in the Karydakis flap group.

In our study, the lengths of hospital stay in the LF and the KF groups were $1.48 \pm 0.50$ and $1.41 \pm 0.49$ days, respectively. Karaca et al. [29] reported that the lengths of hospital stay were 1.1 and 1 days for the Limberg and the Karydakis flap surgeries, respectively. In a study by Bali et al. [23], the durations of hospital stay were 1 and 2 days in the LF and the KF groups, respectively. In addition, the durations of surgery for the Limberg and the Karydakis flap techniques were 29.25 and 22.03 minutes, respectively. In a similar study, the reported durations were, respectively, 50.14 and 42.32 minutes [30]. According to the results of several studies, the duration of Karydakis flap surgery is shorter than the duration of Limburg flap surgery (22-48 minutes vs. $29-54$ minutes) $[20,23,31]$.

The mean score of postoperative pain was approximately similar in patients undergoing Limberg and Karydakis flap surgeries. In a similar study, pain was significantly lower after the Limberg flap surgery $[20,23]$. The present study showed that $14.8 \%$ of patients undergoing Limberg flap surgery had at least one postoperative complication. On the other hand, complications following Karydakis flap surgery were detected in $40.85 \%$ of the patients. The recurrence rate was zero in the Limberg flap group and $2.7 \%$ in the Karydakis flap group.

Bessa [20], in a trial involving 120 patients undergoing a modified Karydakis flap surgery, reported significantly less wound dehiscence compared with patients undergoing a modified Limberg flap surgery (0 patients vs. 9 patients). Patients undergoing the modified Karydakis flap surgery showed, compared to the other group, similar total rates of complications ( $23 \%$ vs. $40 \%$ ), wound infections ( $3 \%$ vs. $5 \%$ ), subcutaneous fluid collection ( $5 \%$ vs. $0 \%$ ), and hypoesthesia (10\% vs. $23 \%)$. However, no significant difference in the rates of recurrence was found between patients treated with the modified Karydakis flap technique and those treated with the modified Limberg flap technique (3\% vs. 2\%). Additionally, Arslan et al. [32], in a trial of 295 patients, found that patients undergoing Karydakis flap surgery had a significantly higher rate of seroma formation compared with patients undergoing Limberg flap or modified Limberg flap surgery (19.8\% vs. $5.2 \%$ vs. $7.4 \%)$. In addition, patients undergoing Karydakis flap surgery had a higher rate of wound dehiscence $(15.4 \%$ vs. $2.1 \%$ vs. $3.7 \%)$ and flap maceration ( $11.0 \%$ vs. $1.0 \%$ vs. $3.7 \%)$.

The relatively short follow-up (6 months) could be one of the limitations of this study, as in several published reports, the follow-up periods were longer than that in the present study. In conclusion, Limberg flap surgery is the standard choice for the treatment of patients with pilonidal disease. Compared to Karydakis flap surgery, it has fewer complications, faster return to work, better overall patient satisfaction, and shorter complete wound healing time.

\section{CONFLICT OF INTEREST}

No potential conflict of interest relevant to this article was reported.

\section{ACKNOWLEDGMENTS}

We would like to express our sincere gratitude to Mazandaran University of Medical Sciences. This study was extracted from the postgraduate thesis of Mojtaba Ghorbani, M.D., which was approved by Mazandaran University of Medical Sciences.

\section{REFERENCES}

1. Khanna A, Rombeau JL. Pilonidal disease. Clin Colon Rectal Surg 2011;24:46-53.

2. Akinci OF, Bozer M, Uzunköy A, Düzgün SA, Coşkun A. Inci- 
dence and aetiological factors in pilonidal sinus among Turkish soldiers. Eur J Surg 1999;165:339-42.

3. Jones DJ. ABC of colorectal diseases. Pilonidal sinus. BMJ 1992; 305:410-2.

4. Hull TL, Wu J. Pilonidal disease. Surg Clin North Am 2002;82: 1169-85.

5. Søndenaa K, Andersen E, Nesvik I, Søreide JA. Patient characteristics and symptoms in chronic pilonidal sinus disease. Int J Colorectal Dis 1995;10:39-42.

6. da Silva JH. Pilonidal cyst: cause and treatment. Dis Colon Rectum 2000;43:1146-56.

7. Kitchen P. Pilonidal sinus - management in the primary care setting. Aust Fam Physician 2010;39:372-5.

8. Davage ON. The origin of sacrococcygeal pilonidal sinuses based on an analysis of four hundred sixty-three cases. Am J Pathol 1954;30:1191-205.

9. Humphries AE, Duncan JE. Evaluation and management of pilonidal disease. Surg Clin North Am 2010;90:113-24.

10. Oncel M, Kurt N, Kement M, Colak E, Eser M, Uzun H. Excision and marsupialization versus sinus excision for the treatment of limited chronic pilonidal disease: a prospective, randomized trial. Tech Coloproctol 2002;6:165-9.

11. Bascom J. Pilonidal disease: long-term results of follicle removal. Dis Colon Rectum 1983;26:800-7.

12. Al-Khamis A, McCallum I, King PM, Bruce J. Healing by primary versus secondary intention after surgical treatment for pilonidal sinus. Cochrane Database Syst Rev 2010;(1):CD006213.

13. Petersen S, Koch R, Stelzner S, Wendlandt TP, Ludwig K. Primary closure techniques in chronic pilonidal sinus: a survey of the results of different surgical approaches. Dis Colon Rectum 2002; 45:1458-67.

14. Nursal TZ, Ezer A, Calişkan K, Törer N, Belli S, Moray G. Prospective randomized controlled trial comparing $\mathrm{V}$-Y advancement flap with primary suture methods in pilonidal disease. Am J Surg 2010;199:170-7.

15. Faux W, Pillai SC, Gold DM. Limberg flap for pilonidal disease: the "no-protractor" approach, 3 steps to success. Tech Coloproctol 2005;9:153-5.

16. Bascom J, Bascom T. Failed pilonidal surgery: new paradigm and new operation leading to cures. Arch Surg 2002;137:1146-50.

17. Bascom J, Bascom T. Utility of the cleft lift procedure in refractory pilonidal disease. Am J Surg 2007;193:606-9.

18. Tezel E. Cleft lift procedure with excision of pits for extensive sacrococcygeal pilonidal disease. Colorectal Dis 2006;8:72.

19. Bessa SS. Results of the lateral advancing flap operation (modified Karydakis procedure) for the management of pilonidal sinus dis- ease. Dis Colon Rectum 2007;50:1935-40.

20. Bessa SS. Comparison of short-term results between the modified Karydakis flap and the modified Limberg flap in the management of pilonidal sinus disease: a randomized controlled study. Dis Colon Rectum 2013;56:491-8.

21. Mentes BB, Leventoglu S, Cihan A, Tatlicioglu E, Akin M, Oguz M. Modified Limberg transposition flap for sacrococcygeal pilonidal sinus. Surg Today 2004;34:419-23.

22. Karydakis GE. Easy and successful treatment of pilonidal sinus after explanation of its causative process. Aust N Z J Surg 1992;62: 385-9.

23. Bali İ, Aziret M, Sözen S, Emir S, Erdem H, Çetinkünar S, et al. Effectiveness of Limberg and Karydakis flap in recurrent pilonidal sinus disease. Clinics (Sao Paulo) 2015;70:350-5.

24. Clothier PR, Haywood IR. The natural history of the post anal (pilonidal) sinus. Ann R Coll Surg Engl 1984;66:201-3.

25. Bchary S. A comparison of prognosis in patients with pilonidal sinus surgery admitted to Shohada hospital of Khorramabad during 2005-2012. Yafteh 2013;15:30-6.

26. Søndenaa K, Pollard ML. Histology of chronic pilonidal sinus. APMIS 1995;103:267-72.

27. Hosseini SV, Rezazadehkermani M, Roshanravan R, Gabash KM, Aghaie-Afshar M. Pilonidal disease: review of recent literature. Ann Colorectal Res 2014;2:e19705. https://doi.org/10.17795/acr19705.

28. Gurer A, Gomceli I, Ozdogan M, Ozlem N, Sozen S, Aydin R. Is routine cavity drainage necessary in Karydakis flap operation? A prospective, randomized trial. Dis Colon Rectum 2005;48:17979.

29. Karaca T, Yoldaş O, Bilgin BÇ, Ozer S, Yoldaş S, Karaca NG. Comparison of short-term results of modified Karydakis flap and modified Limberg flap for pilonidal sinus surgery. Int J Surg 2012; 10:601-6.

30. Ates M, Dirican A, Sarac M, Aslan A, Colak C. Short and longterm results of the Karydakis flap versus the Limberg flap for treating pilonidal sinus disease: a prospective randomized study. Am J Surg 2011;202:568-73.

31. Can MF, Sevinc MM, Hancerliogullari O, Yilmaz M, Yagci G. Multicenter prospective randomized trial comparing modified Limberg flap transposition and Karydakis flap reconstruction in patients with sacrococcygeal pilonidal disease. Am J Surg 2010; 200:318-27.

32. Arslan K, Said Kokcam S, Koksal H, Turan E, Atay A, Dogru O. Which flap method should be preferred for the treatment of pilonidal sinus? A prospective randomized study. Tech Coloproctol 2014;18:29-37. 